\title{
Ergodicity for nonlinear stochastic evolution equations with multiplicative Poisson noise
}

\author{
Carlo Marinelli and Giacomo Ziglio
}

Communicated by Armen Shirikyan, received September 21, 2009.

\begin{abstract}
We study the asymptotic behavior of solutions to stochastic evolution equations with monotone drift and multiplicative Poisson noise in the variational setting, thus covering a large class of (fully) nonlinear partial differential equations perturbed by jump noise. In particular, we provide sufficient conditions for the existence, ergodicity, and uniqueness of invariant measures. Furthermore, under mild additional assumptions, we prove that the Kolmogorov equation associated to the stochastic equation with additive noise is solvable in $L_{1}$ spaces with respect to an invariant measure.
\end{abstract}

\section{Contents}

1. Introduction 2

2. Invariant measures and ergodicity 3

3. Essential $m$-dissipativity of the Kolmogorov operator 12

4. Applications 18

References $\quad 21$

1991 Mathematics Subject Classification. Primary: 60H15, 37A25. Secondary: 60G57, $47 \mathrm{H} 05$.

Key words and phrases. Stochastic PDEs, invariant measures, monotone operators, Kolmogorov equations, Poisson measures.

The authors are sincerely grateful to two anonymous referees for their very careful reading of the first version of this paper. The work for this paper was carried out while the first author was visiting the Department of Statistics of Purdue University supported by a MOIF fellowship. The second author was supported by Project NeSt funded by Provincia Autonoma di Trento and by SFB 701, University of Bielefeld. 


\section{Introduction}

This paper is devoted to the study of asymptotic properties of the solution to an infinite dimensional stochastic differential equation of the type

$$
\left\{\begin{array}{l}
d u(t)+A u(t) d t=\int_{Z} G(u(t-), z) \bar{\mu}(d t, d z) \\
u(0)=x
\end{array}\right.
$$

where $A$ is a nonlinear monotone operator defined on an evolution triple $V \subset$ $H \subset V^{\prime}$ (see e.g. the classical works $[\mathbf{1 7}, \mathbf{2 1}]$ ), and $\bar{\mu}$ is a compensated Poisson measure. Precise assumptions on the data of the problem will be given below. In particular, $A$ may be chosen as the $p$-Laplace operator, as well as the porous media diffusion operator $-\Delta \beta(\cdot)$, thus covering a wide class of nonlinear partial differential equations with discontinuous random perturbations.

While existence and uniqueness of solutions for (1) has been established in [12] (in fact allowing $\bar{\mu}$ to be a general compensated random measure), we are not aware of any result on the asymptotic behavior of the solutions to such equations. Furthermore, as we show in this paper, invariant measures provide a suitable class of reference measures with respect to which one can study infinite dimensional Kolmogorov equations of non-local type, thus extending results that, to the best of our knowledge, were available only for second-order (local) Kolmogorov equations (see e.g. [8]).

Let us briefly describe our main results in more detail: we first prove the existence of an invariant measure for the Markovian semigroup associated to (1), under the (standing) assumption that $V$ is compactly embedded in $H$. Moreover, suitable a priori estimates on any invariant measure imply the existence of an ergodic invariant measure, and an extra superlinearity assumption on $A$ yields exponential mixing, hence uniqueness. Finally, we prove that the (non-local) Kolmogorov operator $L$ associated to (1), with $G$ independent of $u$, is essentially $m$-dissipative in $L_{1}(H, \nu)$, with $\nu$ an infinitesimally invariant measure for $L$. The last result in particular is equivalent to the solvability in $L_{1}(H, \nu)$ of the (elliptic) integro-differential Kolmogorov equation associated to (1).

We should mention that the case where the right-hand side in (1) is replaced by an additive Gaussian noise has been considered in [6], where sufficient conditions for the existence and the uniqueness of invariant measures are given. Moreover, the authors study associated the Kolmogorov equation in $L_{2}(H, \nu)$, assuming that $A$ is differentiable and its differential satisfies a certain polynomial growth condition. Our $L_{1}$ approach does not require any such hypothesis. On the other hand, we need to employ a more sophisticated infinite-dimensional stochastic calculus, which in turns gives rise to a non-local part in the Kolmogorov operator. It is interesting that exactly because of the analytical difficulties created by non-locality we had to adopt the $L_{1}(H, \nu)$ setting, and we could not (better said, we were not able to) use the $L_{2}(H, \nu)$ setting, which is perhaps more natural (see in this respect Lemma 3.1 and Remark 3.4 below). From a qualitative point of view, our results on existence, uniqueness, and ergodicity of invariant measures are perfectly comparable to those of the "classical" case with Wiener noise. Finally, one should mention that combining the results in [6] with ours and appealing to the Lévy-Itô decomposition theorem, one could rather easily obtain corresponding results for evolution equations driven by general (locally) square-integrable Lévy noise. 
In this regard, let us also recall that results on existence and uniqueness of invariant measures for semilinear evolution equations driven by Lévy noise can be found in the recent monograph $[\mathbf{2 3}]$, as well as in $[\mathbf{1 8}]$. However, the authors work in the mild setting, hence equations with fully nonlinear drift (i.e. without a leading linear operator generating a strongly continuous semigroup) cannot be covered. Moreover, ergodicity and polynomial mixing for the complex GinzburgLandau equation (which is also semilinear) driven by a finite intensity Lévy noise have been established in $[\mathbf{2 0}]$.

The rest of the paper is organized as follows: results on existence, uniqueness, and ergodicity of invariant measures $\nu$ are contained in Section 2. In Section 3 , assuming that $G$ does not depend on $u$ and that $A$ satisfies a (mild) "regularizability" hypothesis, we prove that the Kolmogorov operator associated to the stochastic equation (1) is dissipative, hence closable, and its closure is $m$ dissipative in $L_{1}(H, \nu)$. Equivalently, this amounts to saying that the (elliptic) infinite-dimensional non-local Kolmogorov equation associated to (1) is uniquely solvable in $L_{1}(H, \nu)$. In Section 4 we show that our abstract results apply to several situations of interest. In particular, we concentrate on equations with nonlinear drift in divergence form (thus including the $p$-Laplace operator) and on the generalized porous media equations with pure-jump noise.

1.1. Notation. Given a Banach (or Hilbert) space $E$, its norm will be denoted by $|\cdot|_{E}$. We shall denote the space of all Borel measureable bounded functions from $E$ to $\mathbb{R}$ by $B_{b}(E)$. Given another Banach space $F$, the space of $k$-times continuously differentiable functions from $E$ to $F$ will be denoted by $C^{k}(E \rightarrow F)$, with $C^{0}(E \rightarrow F)$ simply denoting the set continuous functions. We shall add a subscript ${ }^{\cdot} b$ if the functions themselves and all their derivatives (up to order $k$ ) are bounded. If $\phi: E \rightarrow F$ is Lipschitz continuous, we shall write $\phi \in \dot{C}^{0,1}(E \rightarrow F)$, and we define

$$
|\phi|_{\dot{C}^{0,1}(E \rightarrow F)}:=\sup _{x, y \in E, x \neq y} \frac{|\phi(x)-\phi(y)|_{F}}{|x-y|_{E}} .
$$

If $F=\mathbb{R}$, we shall simply write $C^{k}(E)$ etc. Sometimes we shall just write $C^{k}$ etc. if it is obvious what $E$ and $F$ are. By $\mathcal{M}_{1}(E)$ we shall indicate the space of probability measures on $E$, endowed with the $\sigma\left(\mathcal{M}_{1}(E), C_{b}^{0}(E)\right)$ topology induced by duality with bounded continuous functions, and usually known as the topology of weak convergence. Weak convergence (of functions and measures) will be denoted by $\rightarrow$, without explicit reference to the underlying topology if no confusion may arise.

If $X \leq N Y$ for some positive constant $N$, we shall equivalently write $X \lesssim Y$. If $N$ depends on a set of parameters $p_{1}, \ldots, p_{n}$, we shall also write $N=N\left(p_{1}, \ldots, p_{n}\right)$ and $X \lesssim_{p_{1}, \ldots, p_{n}} Y$.

\section{Invariant measures and ergodicity}

Let $\left(\Omega, \mathcal{F},\left(\mathcal{F}_{t}\right)_{t \geq 0}, \mathbb{P}\right)$ be a filtered probability space satisfying the "usual hypotheses" (see e.g. [19, Definition 1.1]), and $\mathbb{E}$ denote expectation with respect to $\mathbb{P}$. All stochastic elements will be defined on this stochastic basis, unless otherwise specified. Let $(Z, \mathcal{Z}, m)$ be a measure space with a $\sigma$-finite measure $m$ and $\mu$ a Poisson random measure on $\mathbb{R}_{+} \times Z$ with compensator Leb $\otimes m$, and set $\bar{\mu}:=\mu-\operatorname{Leb} \otimes m$ (Leb stands for Lebesgue measure on $\mathbb{R}$ ). For a comprehensive account of stochastic integration with respect to compensated Poisson random measure see e.g. [19, 
$\S 31]$, or [15]. Let $H$ be a real separable Hilbert space, and $G: H \times Z \rightarrow H$ a measurable function such that

$$
|G(x, \cdot)|_{m}^{2}:=\int_{Z}|G(x, z)|_{H}^{2} m(d z)<\infty \quad \forall x \in H .
$$

Let $V$ and $V^{\prime}$ be a reflexive Banach space and its dual, respectively, such that $V \hookrightarrow$ $H \hookrightarrow V^{\prime}$ with dense and continous embeddings. Thanks to Asplund's renorming theorem [3], we shall assume without loss of generality that both $V$ and $V^{\prime}$ are strictly convex. Furthermore, we shall assume that $V \hookrightarrow H$ is compact. Both the duality pairing between $V$ and $V^{\prime}$ and the inner product in $H$ will be denoted by $\langle\cdot, \cdot\rangle$.

The operator $A: V \rightarrow V^{\prime}$ is assumed to be demicontinuous (i.e. stronglyweakly closed) and to satisfy the monotonicity condition

$$
2\langle A x-A y, x-y\rangle-|G(x, \cdot)-G(y, \cdot)|_{m}^{2} \geq 0 \quad \forall x, y \in V,
$$

as well as the following coercivity and growth conditions:

$$
\begin{aligned}
2\langle A x, x\rangle-|G(x, \cdot)|_{m}^{2}+\alpha_{0}|x|_{H}^{2} & \geq \alpha_{1}|x|_{V}^{p}-C_{0} & & \forall x \in V, \\
|A x|_{V^{\prime}} & \leq C_{1}|x|_{V}^{p-1}+C_{2} & & \forall x \in V,
\end{aligned}
$$

for some constants $\alpha_{0} \geq 0, \alpha_{1}>0, C_{0}, C_{1}>0, C_{2} \in \mathbb{R}$ and $p>2$. Instead of (3) one could assume that there exists a constant $\alpha_{1}>0$ such that

$$
2\langle A x, x\rangle-|G(x, \cdot)|_{m}^{2} \geq \alpha_{1}|x|_{V}^{2} \quad \forall x \in V .
$$

Note that, by (3) and (4), one has

$$
|G(x, \cdot)|_{m}^{2} \leq 2 C_{1}|x|_{V}^{p}+\alpha_{0}|x|_{H}^{2}+2 C_{2}|x|_{V}+C_{0} \quad \forall x \in V .
$$

All assumptions stated so far will be in force throughout the paper and will be used without further mention.

Let us recall the following well-posedness result for (1) due to Gyöngy [12, Thm. 2.10]. Here and in the following we shall denote the space of $H$-valued random variables with finite $p$-th moment by $\mathbb{L}_{p}(H)$, and the space of adapted processes $X:[0, T] \rightarrow H$ such that $\mathbb{E} \sup _{t \leq T}|X(t)|_{H}^{p}<\infty$ by $\mathbb{H}_{p}(T)$.

Proposition 2.1. Let $x \in \mathbb{L}_{2}(H)$ and $T \geq 0$. Then equation (1) admits a unique strong solution $u$ such that $u(t) \in V \mathbb{P}$-a.s. for a.a. $t \in[0, T], t \mapsto u(t)$ is càdlàg in $H$, and satisfies

$$
\mathbb{E} \sup _{t \leq T}|u(t)|_{H}^{2}+\mathbb{E} \int_{0}^{T}|u(t)|_{V}^{p} d t<\infty .
$$

Moreover, $u$ is a Markov process, and the solution map $x \mapsto u$ is Lipschitz continuous from $\mathbb{L}_{2}$ to $\mathbb{H}_{2}(T)$.

The solution to (1) generates a Markovian semigroup $P_{t}$ on $B_{b}(H)$ by the usual prescription $P_{t} \phi(x)=\mathbb{E} \phi(u(t, x)), \phi \in B_{b}(H)$, where $u(\cdot, x)$ denotes the solution to (1). The continuity of the solution map ensures that $P_{t}$ is Feller, i.e. $\phi \in C_{b}^{0}(H)$ implies $P_{t} \phi \in C_{b}^{0}(H)$ for all $t \geq 0$ (see e.g. [8, $\left.\S 1.2 .5\right]$ ).

In the following subsection we establish the existence and uniqueness of an ergodic invariant measure for $P_{t}$ under an assumption stronger than (2). The proof is adapted from a classical method used for stochastic evolution equations with Wiener noise in the mild setting (see e.g. [8, §3.4]). This simple result is included only for completeness, while the main results of this section are contained in $§ 2.2$. 
2.1. Strictly dissipative case. Throughout this subsection we assume that there exists $\alpha \in(0, \infty)$ such that

$$
2\langle A x-A y, x-y\rangle-|G(x, \cdot)-G(y, \cdot)|_{m}^{2} \geq \alpha|x-y|_{H}^{2} \quad \forall x, y \in V .
$$

We shall need a few preparatory results. The following inequality can be obtained by a simple computation based on (5), (6) and Young's inequality (see e.g. [24, $\S 4.3]$ for a related case).

Lemma 2.2. Let $\eta \in(0, \alpha)$. There exist $\delta_{\eta} \in(0, \infty)$ such that

$$
2\langle A x, x\rangle-|G(x, \cdot)|_{m}^{2} \geq \eta|x|_{H}^{2}-\delta_{\eta} \quad \forall x \in V .
$$

Let us define the random measure $\mu_{1}$ on $\mathbb{R} \times Z$ as

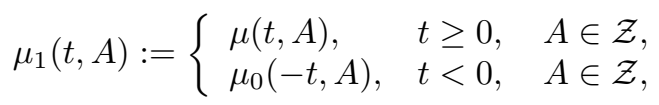

with $\mu_{0}$ an independent copy of $\mu$, on the naturally associated filtration $\left(\tilde{\mathcal{F}}_{t}\right)_{t \in \mathbb{R}}$. Let us also define the compensated measure measure $\tilde{\mu}:=\mu_{1}-$ Leb $\otimes m$.

For $s \in \mathbb{R}$, consider the equation

$$
\left\{\begin{array}{l}
d u(t)+A u(t) d t=\int_{Z} G(u(t-), z) \tilde{\mu}(d t, d z), \quad t \geq s \\
u(s)=x
\end{array}\right.
$$

It is clear that (8) admits a unique solution $u(t, s, x)$ which generates a semigroup $P_{s, t}$ on $B_{b}(H)$, exactly as above.

Lemma 2.3. Let $s \in(-\infty, 0]$ and $x \in \mathbb{L}_{2}(H)$. There exists $v \in \mathbb{L}_{2}(H)$, independent of $x$, such that

$$
\lim _{s \rightarrow-\infty} \mathbb{E}|u(0, s, x)-v|_{H}^{2}=0
$$

Moreover, one has

$$
\mathbb{E}|u(0, s, x)-v|_{H}^{2} \lesssim e^{\alpha s}\left(1+\mathbb{E}|x|_{H}^{2}\right)
$$

Proof. For $s_{1}, s_{2} \in(-\infty, 0], s_{1} \leq s_{2}$ and $x \in \mathbb{L}_{2}(H)$, we have

$$
\begin{aligned}
u\left(0, s_{1}, x\right)-u\left(0, s_{2}, x\right)= & -\int_{s_{2}}^{0}\left[A u\left(r, s_{1}, x\right)-A u\left(r, s_{2}, x\right)\right] d r \\
& +\int_{s_{2}}^{0} \int_{Z}\left[G\left(u\left(r, s_{1}, x\right), z\right)-G\left(u\left(r, s_{2}, x\right), z\right)\right] \tilde{\mu}(d r, d z) \\
& +u\left(s_{2}, s_{1}, x\right)-x .
\end{aligned}
$$

Appealing to Itô's formula for the square of the norm (see [14]), and recalling (6), we obtain

$$
\begin{aligned}
\mathbb{E}\left|u\left(0, s_{1}, x\right)-u\left(0, s_{2}, x\right)\right|_{H}^{2} \\
\leq-2 \mathbb{E} \int_{s_{2}}^{0}\left\langle A\left(u\left(r, s_{1}, x\right)-u\left(r, s_{2}, x\right)\right), u\left(r, s_{1}, x\right)-u\left(r, s_{2}, x\right)\right\rangle d r \\
\quad+\mathbb{E} \int_{s_{2}}^{0}\left|G\left(u\left(r, s_{1}, x\right), \cdot\right)-G\left(u\left(r, s_{2}, x\right), \cdot\right)\right|_{m}^{2} d r+\mathbb{E}\left|u\left(s_{2}, s_{1}, x\right)-x\right|_{H}^{2} \\
\leq 2 e^{\alpha s_{2}}\left[\mathbb{E}\left|u\left(s_{2}, s_{1}, x\right)\right|_{H}^{2}+\mathbb{E}|x|_{H}^{2}\right]
\end{aligned}
$$


as well as, recalling (7),

$$
\mathbb{E}\left|u\left(s_{2}, s_{1}, x\right)\right|_{H}^{2} \leq\left(\frac{\delta_{\eta}}{\eta}+\mathbb{E}|x|_{H}^{2}\right) e^{\eta\left(s_{1}-s_{2}\right)} \leq\left(\frac{\delta_{\eta}}{\eta}+\mathbb{E}|x|_{H}^{2}\right) .
$$

Combining these inequalities we obtain

$$
\mathbb{E}\left|u\left(0, s_{1}, x\right)-u\left(0, s_{2}, x\right)\right|_{H}^{2} \leq 2\left(\frac{\delta_{\eta}}{\eta}+2 \mathbb{E}|x|_{H}^{2}\right) e^{\alpha s_{2}} .
$$

Letting $s_{2}$ tend to $-\infty$, it follows that there exists $v(x) \in \mathbb{L}_{2}(H)$ such that

$$
\lim _{s \rightarrow-\infty} \mathbb{E}|u(0, s, x)-v(x)|_{H}^{2}=0 .
$$

By the same arguments one can prove that

$$
\lim _{s \rightarrow-\infty} \mathbb{E}|u(0, s, x)-u(0, s, y)|_{H}^{2}=0
$$

for all $x, y \in \mathbb{L}_{2}(H)$, hence that $v$ is independent of $x \in \mathbb{L}_{2}(H)$. Letting $s_{1}$ tend to $-\infty$ in (9) one obtains the exponential convergence.

We can now prove the main result of this subsection.

TheOREm 2.4. Assume that (6) holds and that $x \in \mathbb{L}_{2}(H)$. There exists a unique invariant measure $\nu$ for the semigroup $P_{t}$ associated to (1). Moreover, one has

$$
\int|y|_{H}^{2} \nu(d y)<\infty
$$

and

$$
\left|P_{t} \varphi(y)-\int \varphi(w) \nu(d w)\right| \leq e^{-\frac{\alpha}{2} t}|\varphi|_{\dot{C}^{0,1}(H)} \int|y-w|_{H} \nu(d w)
$$

for all $t \geq 0, y \in H$ and $\varphi: H \rightarrow \mathbb{R}$ bounded and Lipschitz.

Proof. Let $\nu$ be the law of the random variable $v$ constructed in Lemma 2.3. In particular, $\int|y|^{2} \nu(d y)<\infty$ is equivalent to $v \in \mathbb{L}_{2}(H)$. Similarly, the previous lemma immediately yields $P_{s, 0}^{*} \delta_{y} \rightarrow \nu$ for all $y \in H$ in $\mathcal{M}_{1}(H)$ as $s \rightarrow-\infty$. Moreover, for any $\varphi: H \rightarrow \mathbb{R}$ Lipschitz continuous and bounded, we have

$$
\int\left(P_{0, t} \varphi\right) d \nu=\lim _{s \rightarrow-\infty} \int\left(P_{0, t} \varphi\right) d\left(P_{s, 0}^{*} \delta_{y}\right)=\lim _{s \rightarrow-\infty}\left(P_{s-t, 0} \varphi\right)(y)=\int \varphi d \nu
$$

i.e. $\nu$ is invariant for $P_{t}$. Moreover, if $\nu$ is an invariant measure for $P_{t}$,

$$
\left|P_{t} \varphi(y)-\int \varphi d \nu\right|=\left|\int\left(P_{t} \varphi(y)-P_{t} \varphi(w)\right) \nu(d w)\right| \leq e^{-\frac{\alpha}{2} t}|\varphi|_{\dot{C}^{0,1}(H)} \int|y-w|_{H} \nu(d w)
$$

for all $t \geq 0$. In fact, by the same arguments used to derive (9), we get

$$
\mathbb{E}|u(t, y)-u(t, w)|_{H}^{2} \leq e^{-\alpha t}|y-w|_{H}^{2}
$$

and

$$
\begin{aligned}
\left|\int\left(P_{t} \varphi(y)-P_{t} \varphi(w)\right) \nu(d w)\right| & \leq|\varphi|_{\dot{C}^{0,1}(H)} \int \mathbb{E}|u(t, y)-u(t, w)|_{H} \nu(d w) \\
& \leq|\varphi|_{\dot{C}^{0,1}(H)} \int\left(\mathbb{E}|u(t, y)-u(t, w)|_{H}^{2}\right)^{\frac{1}{2}} \nu(d w) \\
& \leq e^{-\frac{\alpha}{2} t}|\varphi|_{\dot{C}^{0,1}(H)} \int|y-w|_{H} \nu(d w) .
\end{aligned}
$$


Let us prove uniqueness of $\nu$. Let $\nu^{\prime}$ be an invariant measure for $P_{t}$, i.e.

$$
\int_{H} P_{t} \varphi(y) \nu^{\prime}(d y)=\int_{H} \varphi(y) \nu^{\prime}(d y)
$$

for all $\varphi \in C_{b}^{0}(H)$. Then, letting $t$ tend to $+\infty$, since

$$
\lim _{t \rightarrow+\infty} P_{t} \varphi(y)=\int_{H} \varphi d \nu
$$

for all $y \in H, \varphi \in C_{b}^{0}(H)$, we obtain, by the dominated convergence theorem,

$$
\int_{H}\left(\int_{H} \varphi d \nu\right) \nu^{\prime}(d y)=\int_{H} \varphi d \nu=\int_{H} \varphi d \nu^{\prime}
$$

for all $\varphi \in C_{b}^{0}(H)$, i.e. $\nu=\nu^{\prime}$.

2.2. General case. We can still prove the existence of an ergodic invariant measure without the assumption that the couple $(A, G)$ is strictly dissipative, using an argument based on Krylov-Bogoliubov's theorem (see e.g. [8, Thm. 1.11]).

TheOREM 2.5. There exists an invariant measure $\nu$ for $P_{t}$. Moreover, $\nu$ is concentrated on $V$, i.e. $\nu(V)=1$.

Proof. We assume $p>2$, since the proof for the case $p=2$ is completely similar. Let $x \in \mathbb{L}_{2}(H)$. By Itô's formula for the square of the norm in $H$ (see [14]) we have

$$
\begin{aligned}
|u(t, x)|_{H}^{2}- & |x|_{H}^{2}=2 \int_{0}^{t}\langle u(s-, x), d u(s, x)\rangle+[u](t) \\
= & -2 \int_{0}^{t}\langle A u(s, x), u(s, x)\rangle d s+2 \int_{0}^{t} \int_{Z}\langle u(s-, x), G(u(s-, x), z)\rangle \bar{\mu}(d s, d z) \\
& +\int_{0}^{t} \int_{Z}|G(u(s-, x), z)|_{H}^{2} \mu(d s, d z),
\end{aligned}
$$

where $[u]$ stands for the quadratic variation of $u$ (see e.g. $[\mathbf{1 9}, \S 25]$ ). Taking expectations on both side and recalling that the compensator of $\mu$ is Leb $\otimes m$, we obtain

$$
\mathbb{E}|u(t, x)|_{H}^{2}=-2 \mathbb{E} \int_{0}^{t}\langle A u(s, x), u(s, x)\rangle d s+\mathbb{E}|x|_{H}^{2}+\int_{0}^{t}|G(u(s, x), \cdot)|_{m}^{2} d s,
$$

hence, thanks to $(3)$,

$$
\mathbb{E}|u(t, x)|_{H}^{2} \leq \alpha_{0} \mathbb{E} \int_{0}^{t}|u(s, x)|_{H}^{2} d s-\alpha_{1} \mathbb{E} \int_{0}^{t}|u(s, x)|_{V}^{p} d s+\mathbb{E}|x|_{H}^{2}+t C_{0} .
$$

Since $V \hookrightarrow H$ is continuous, there exists a constant $c>0$ such that $|v|_{H} \leq c|v|_{V}$ for all $v \in V$, hence

$$
\mathbb{E}|u(t, x)|_{H}^{2} \leq \alpha_{0} \mathbb{E} \int_{0}^{t}|u(s, x)|_{H}^{2} d s-\frac{\alpha_{1}}{c^{p}} \mathbb{E} \int_{0}^{t}|u(s, x)|_{H}^{p} d s+\mathbb{E}|x|_{H}^{2}+t C_{0} .
$$

The elementary inequality $\varepsilon^{2}|y|^{2} \leq \varepsilon^{p}|y|^{p}+1$ (with $\varepsilon>0$ and $p \geq 2$ ) yields

$$
-\mathbb{E}|u(t, x)|_{H}^{p} \leq-\varepsilon^{2-p} \mathbb{E}|u(t, x)|_{H}^{2}+\varepsilon^{-p},
$$


thus also

$$
\begin{aligned}
\mathbb{E}|u(t, x)|_{H}^{2} & \leq-\left(\frac{\alpha_{1} \varepsilon^{2-p}}{c^{p}}-\alpha_{0}\right) \int_{0}^{t} \mathbb{E}|u(s, x)|_{H}^{2} d s+t\left(\frac{\alpha_{1} \varepsilon^{-p}}{c^{p}}+C_{0}\right)+\mathbb{E}|x|_{H}^{2} \\
& =-\gamma \int_{0}^{t} \mathbb{E}|u(s, x)|_{H}^{2} d s+\mathbb{E}|x|_{H}^{2}+t C
\end{aligned}
$$

where

$$
\gamma:=\frac{\alpha_{1} \varepsilon^{2-p}}{c^{p}}-\alpha_{0}, \quad C:=\frac{\alpha_{1} \varepsilon^{-p}}{c^{p}}+C_{0} .
$$

Choosing $\varepsilon$ so that $\gamma>0$ and applying Gronwall's inequality to (12), it follows that

$$
\mathbb{E}|u(t, x)|_{H}^{2} \leq \mathbb{E}|x|_{H}^{2} e^{-\gamma t}+K \quad \forall t \geq 0
$$

where $K$ is a constant independent of $t$. Moreover, by (11) and (13) we obtain

$$
\begin{aligned}
\mathbb{E} \int_{0}^{t}|u(s, x)|_{V}^{p} d s & \leq \frac{1}{\alpha_{1}}\left(\alpha_{0} \mathbb{E} \int_{0}^{t}|u(s, x)|_{H}^{2} d s+\mathbb{E}|x|_{H}^{2}+t C_{0}\right) \\
& \leq \frac{1}{\alpha_{1}}\left[\left(\frac{\alpha_{0}}{\gamma}+1\right) \mathbb{E}|x|_{H}^{2}+t\left(\alpha_{0} K+C_{0}\right)\right]
\end{aligned}
$$

for all $t>0$.

We shall now use the estimates just obtained to prove the tightness of the sequence of measures

$$
\nu_{n}:=\frac{1}{n} \int_{0}^{n} \lambda_{t} d t, \quad n \in \mathbb{N},
$$

where $\lambda_{t}$ stands for the law of the random variable $u(t, 0)$, so that

$$
\int_{H} \varphi d \nu_{n}=\frac{1}{n} \int_{0}^{n} \mathbb{E} \varphi(u(t, 0)) d t
$$

for all $\varphi \in B_{b}(H)$. By (14) we obtain

$$
\mathbb{E} \int_{0}^{t}|u(s, 0)|_{V}^{p} d s \lesssim t \quad \forall t>0,
$$

which in turn implies

$$
\int_{H}|y|_{V}^{p} \nu_{n}(d y)=\frac{1}{n} \int_{0}^{n} \mathbb{E}|u(s, 0)|_{V}^{p} d s \lesssim 1 \quad \forall n \in \mathbb{N} .
$$

By Markov's inequality we thus obtain

$$
\sup _{n \in \mathbb{N}} \nu_{n}\left(|y|_{V} \geq R\right) \leq \sup _{n \in \mathbb{N}} \frac{1}{n R^{p}} \int_{0}^{n} \mathbb{E}|u(s, 0)|_{V}^{p} d s \lesssim \frac{1}{R^{p}},
$$

which converges to zero as $R \rightarrow \infty$. Since the ball $B_{R}:=\left\{y \in H:|y|_{V} \leq R\right\}$ is bounded in $V$, and $V \hookrightarrow H$ is compact, it follows that, for any given $\varepsilon$, there exists $\bar{R} \in \mathbb{R}_{+}$such that $\nu_{n}\left(B_{\bar{R}}\right)>1-\varepsilon$ uniformly over $n$, with $B_{\bar{R}}$ a compact subset of $H$. In other words, the sequence $\nu_{n}$ is tight, and Prohorov's theorem (see e.g. $[\mathbf{1}, \S 15.5])$ yields the existence of a subsequence $\nu_{n_{k}}$ such that $\nu_{n_{k}} \rightarrow \nu$ in $\mathcal{M}_{1}(H)$. Furthermore, recalling that $P_{t}$ is Feller on $H, \nu$ is an invariant measure for $P_{t}$ by Krylov-Bogoliubov's theorem.

Let us now show that $\nu$ is concentrated on $V$. To this end, let us define the function $\Theta(y):=\sup _{k \in \mathbb{N}}\left|\left\langle\ell_{k}, y\right\rangle\right|$, where $\left\{\ell_{k}\right\}_{k \in \mathbb{N}}$ is a countable dense subset of $B_{1}^{V^{\prime}} \cap H$ in the topology of $H$, and $B_{1}^{V^{\prime}}$ is the closed unit ball in $V^{\prime}$. Hence it 
follows (e.g. by $[\mathbf{2 4}$, p. 74]) that $\Theta: H \rightarrow[0, \infty]$ is a lower semicontinuous function such that

$$
\Theta(y)= \begin{cases}|y|_{V}, & y \in V \\ +\infty, & y \in H \backslash V\end{cases}
$$

Then (15) implies

$$
\begin{aligned}
\int_{H} \Theta(y)^{p} \nu(d y) & =\lim _{L \rightarrow \infty} \lim _{M \rightarrow \infty} \int_{H}\left(\sup _{k \leq L}\left|\left\langle\ell_{k}, y\right\rangle\right|^{p} \wedge M\right) \nu(d y) \\
& =\sup _{L, M \in \mathbb{N}} \lim _{h \rightarrow \infty} \int_{H}\left(\sup _{k \leq L}\left|\left\langle\ell_{k}, y\right\rangle\right|^{p} \wedge M\right) \nu_{n_{h}}(d y) \\
& \leq \liminf _{h \rightarrow \infty} \sup _{L, M \in \mathbb{N}} \int_{H}\left(\sup _{k \leq L}\left|\left\langle\ell_{k}, y\right\rangle\right|^{p} \wedge M\right) \nu_{n_{h}}(d y) \\
& =\liminf _{h \rightarrow \infty} \int_{H}|y|_{V}^{p} \nu_{n_{h}}(d y)<\infty,
\end{aligned}
$$

hence $\Theta<\infty \nu$-a.e., thus also $\nu(V)=1$ since $\{y \in H: \Theta(y)<\infty\}=V$.

THEOREM 2.6. Let $\nu$ be an invariant measure for $P_{t}$. Then $\nu$ satisfies the estimate

$$
\int_{H}\left(|x|_{H}^{2}+|x|_{V}^{p}\right) \nu(d x)<\infty .
$$

Proof. Let $x \in H$ and consider the one dimensional process $U(t):=|u(t, x)|_{H}^{2}$, which can be written, in view of (10), as

$$
U(t)=|x|_{H}^{2}+\int_{0}^{t} F_{1}(s) d s+\int_{0}^{t} \int_{Z} F_{2}(s, z) \bar{\mu}(d s, d z)+\int_{0}^{t} \int_{Z} F_{3}(s, z) \mu(d s, d z),
$$

where $F_{1}, F_{2}, F_{3}$ are defined in the obvious way. Let $\chi \in C_{b}^{1}\left(\mathbb{R}_{+}, \mathbb{R}\right)$ be a smooth cutoff function with $\chi(x)=1$ for all $x \in[0,1], \chi(x)=0$ for all $x \geq 2$, and $\chi^{\prime}(x) \leq 0$ for all $x \in \mathbb{R}_{+}$. Setting $\chi_{N}(x)=\chi(x / N)$ and $\varphi_{N}(x)=\int_{0}^{x} \chi_{N}(y) d y$ for all $x \in \mathbb{R}_{+}$, Itô's formula yields, suppressing the $\cdot_{H}$ subscript for simplicity of notation,

$$
\begin{aligned}
\varphi_{N}(U(t))= & \varphi_{N}\left(|x|^{2}\right)+\int_{0}^{t} \varphi_{N}^{\prime}(U(s-)) d U(s) \\
& +\sum_{s \leq t}\left[\varphi_{N}(U(s-)+\Delta U(s))-\varphi_{N}(U(s-))-\varphi_{N}^{\prime}(U(s-)) \Delta U(s)\right]
\end{aligned}
$$

By Taylor's formula, there exists $\theta \in(0,1)$ such that the summand in the last term on the right-hand side can be written as

$$
\frac{1}{2} \varphi_{N}^{\prime \prime}(U(s-)+\theta \Delta U(s))|\Delta U(s)|^{2},
$$

which is negative $\mathbb{P}$-a.s. because $\varphi_{N}^{\prime \prime}(x)=\chi_{N}^{\prime}(x)=N^{-1} \chi^{\prime}(x / N) \leq 0$ for all $x \in \mathbb{R}_{+}$. Moreover, the second term on the right-hand side of (16) can be written as

$$
\begin{gathered}
-2 \int_{0}^{t} \chi_{N}\left(|u(s)|^{2}\right)\langle A u(s), u(s)\rangle d s+2 \int_{0}^{t} \int_{Z} \chi_{N}\left(|u(s-)|^{2}\right) \\
\langle u(s-), G(u(s-), z)\rangle \bar{\mu}(d s, d z)+\int_{0}^{t} \int_{Z} \chi_{N}\left(|u(s-)|^{2}\right)|G(u(s-), z)|^{2} \mu(d s, d z) .
\end{gathered}
$$


Therefore, taking expectation on both sides of (16), recalling that the compensator of $\mu$ is Leb $\otimes m$, we are left with

$$
\begin{aligned}
\mathbb{E} \varphi_{N}\left(|u(t)|^{2}\right) \leq & \mathbb{E} \varphi_{N}\left(|x|^{2}\right)-2 \mathbb{E} \int_{0}^{t} \chi_{N}\left(|u(s)|^{2}\right)\langle A u(s), u(s)\rangle d s \\
& +\mathbb{E} \int_{0}^{t} \int_{Z} \chi_{N}\left(|u(s)|^{2}\right)|G(u(s), z)|^{2} m(d z) d s .
\end{aligned}
$$

Recalling (3), Tonelli's theorem yields

$$
\begin{aligned}
& \mathbb{E} \varphi_{N}\left(|u(t)|^{2}\right)+\alpha_{1} \int_{0}^{t} \mathbb{E} \chi_{N}\left(|u(s)|^{2}\right)|u(s)|_{V}^{p} d s \\
& \leq \mathbb{E} \varphi_{N}\left(|x|^{2}\right)+\alpha_{0} \int_{0}^{t} \mathbb{E} \chi_{N}\left(|u(s)|^{2}\right)|u(s)|^{2} d s+t C_{0} .
\end{aligned}
$$

for all $t \geq 0$. Integrating both sides with respect to $\nu$ on $H$, applying again Tonelli's theorem, the definition of invariant measure, and setting $t=1$, we obtain

$$
\alpha_{1} \int_{H} \chi_{N}\left(|x|^{2}\right)|x|_{V}^{p} \nu(d x) \leq \alpha_{0} \int_{H} \chi_{N}\left(|x|^{2}\right)|x|^{2} \nu(d x)+C_{0} .
$$

By the inequality $\varepsilon^{2}|x|^{2} \leq \varepsilon^{p}|x|^{p}+1$ and the continuity of $V \hookrightarrow H$, we have

$$
\int_{H} \chi_{N}\left(|x|^{2}\right)|x|^{2} \nu(d x) \leq \varepsilon^{p-2} c^{p} \int_{H} \chi_{N}\left(|x|^{2}\right)|x|_{V}^{p} \nu(d x)+\varepsilon^{-2},
$$

hence

$$
\int_{H} \chi_{N}\left(|x|^{2}\right)|x|^{2} \nu(d x) \leq \varepsilon^{-2}+\frac{\varepsilon^{p-2} c^{p}}{\alpha_{1}}\left(\alpha_{0} \int_{H} \chi_{N}\left(|x|^{2}\right)|x|^{2} \nu(d x)+C_{0}\right) .
$$

Choosing $\varepsilon$ sufficiently small we get

$$
\int_{H} \chi_{N}\left(|x|^{2}\right)|x|^{2} \nu(d x) \lesssim 1
$$

thus also, by the monotone convergence theorem, $\int_{H}|x|^{2} \nu(d x)<\infty$. This immediately yields the result, in view of (17).

The estimates just established allow one to deduce the existence of an ergodic invariant measure.

COROLlaRY 2.7. There exists an ergodic invariant measure for the semigroup $P_{t}$.

Proof. The last estimate in the proof of the previous theorem and (17) allow to conclude that there exists a constant $N$, independent of $\nu$, such that

$$
\int_{H}|x|_{V}^{p} \nu(d x)<N
$$

for any invariant measure $\nu$. Denoting by $\mathcal{N} \subset \mathcal{M}_{1}(H)$ the set of invariant measures of $P_{t}$, Markov's inequality yields

$$
\sup _{\nu \in \mathcal{N}} \nu\left(|x|_{V}>R\right) \leq \frac{1}{R^{p}} \sup _{\nu \in \mathcal{N}} \int_{H}|x|_{V}^{p} \nu(d x)<\frac{N}{R^{p}} \stackrel{R \rightarrow+\infty}{\longrightarrow} 0 .
$$

Therefore, by the same argument used in the proof of Theorem 2.5, we conclude that $\mathcal{N}$ is tight, hence, thanks to Prohorov's theorem, (relatively) compact in $\mathcal{M}_{1}(H)$. 
Since $\mathcal{N}$ is non-empty and convex, Krein-Milman's theorem ensures that $\mathcal{N}$ has extreme points, which are ergodic invariant measures for $P_{t}$ by a well-known criterion (see e.g. [1, thm. 19.25]).

Finally, we give a sufficient condition for uniqueness of an invariant measure under an extra superlinearity assumption on the couple $(A, G)$.

Proposition 2.8. Assume that there exist $\eta>0$ and $\delta>0$ such that

$$
2\langle A v-A w, v-w\rangle-|G(v, \cdot)-G(w, \cdot)|_{m}^{2} \geq \eta|v-w|_{H}^{2+\delta}, \quad \forall v, w \in V .
$$

Then $P_{t}$ has a unique strongly mixing invariant measure.

Proof. Let $x, y \in H$. Then Itô's formula for the square of the norm in $H$ implies, after taking expectations,

$$
\begin{aligned}
\mathbb{E}|u(t, x)-u(t, y)|^{2} & +2 \mathbb{E} \int_{0}^{t}\langle A u(s, x)-A u(s, y), u(s, x)-u(s, y)\rangle d s \\
& =|x-y|^{2}+\mathbb{E} \int_{0}^{t} \int_{Z}|G(u(s, x), z)-G(u(s, y), z)|^{2} m(d z) d s,
\end{aligned}
$$

and, by the superlinearity assumption (18),

$$
\begin{aligned}
\mathbb{E}|u(t, x)-u(t, y)|^{2} & \leq|x-y|^{2}-\eta \int_{0}^{t} \mathbb{E}|u(s, x)-u(s, y)|^{2+\delta} d s \\
& \leq|x-y|^{2}-\eta \int_{0}^{t}\left(\mathbb{E}|u(s, x)-u(s, y)|^{2}\right)^{1+\delta / 2} d s
\end{aligned}
$$

for all $t>0$, where we have used Jensen's inequality in the last step. Since the solution $\zeta: \mathbb{R}_{+} \rightarrow \mathbb{R}_{+}$of the ordinary differential equation

$$
\zeta^{\prime}=-\eta \zeta^{1+\delta / 2}, \quad \zeta(0)=|x-y|^{2}
$$

is such that $\lim _{t \rightarrow \infty} \zeta(t)=0$ for all $x, y \in H$, we conclude by a standard comparison argument that $\mathbb{E}|u(t, x)-u(t, y)|^{2} \rightarrow 0$ as $t \rightarrow \infty$.

Let $\nu$ be an invariant measure for $P_{t}$. Then for any Lipschitz continuous and bounded $f: H \rightarrow \mathbb{R}$ we have

$$
\begin{aligned}
\left|P_{t} f(x)-\int_{H} f d \nu\right| & =\left|\int_{H} P_{t} f(x) \nu(d y)-\int_{H} P_{t} f(y) \nu(d y)\right| \\
& \leq \int_{H}\left|P_{t} f(x)-P_{t} f(y)\right| \nu(d y) \\
& \leq|f|_{\dot{C}^{0,1}(H)} \int_{H}\left(\mathbb{E}|u(t, x)-u(t, y)|^{2}\right)^{1 / 2} \nu(d y) .
\end{aligned}
$$

Since $\left(\mathbb{E}|u(t, x)-u(t, y)|^{2}\right)^{1 / 2} \leq|x-y|$ and $\int_{H}|x-y| \nu(d y)<\infty$, we can pass to the limit under the integral sign as $t \rightarrow \infty$ by the dominated convergence theorem, thus concluding that $\left|P_{t} f(x)-\int_{H} f d \nu\right| \rightarrow 0$ as $t \rightarrow \infty$, and in particular that $\nu$ is the unique invariant measure. Moreover, since $C_{b}^{1}(H)$ is dense in $L_{2}(H, \nu)$, one has that for any $f \in L_{2}(H, \nu)$,

$$
\lim _{t \rightarrow \infty} P_{t} f(x)=\int_{H} f d \nu, \quad x \in H,
$$

i.e. $\nu$ is strongly mixing (in particular ergodic) as required. 


\section{Essential $m$-dissipativity of the Kolmogorov operator}

We recall that in this section we will work under the assumption that the noise in (1) is additive, i.e. $G(x, z)=G(z)$. Unfortunately we are not able, at the moment, to consider Kolmogorov equations associated to stochastic equations with multiplicative noise, as several highly non-trivial complications arise. The situation is not different in the context of equations with Wiener noise, where results on "multiplicative" Kolmogorov equations have been obtained only as perturbations of the infinite-dimensional heat equation, or assuming very regular coefficients (typically of class $C_{b}^{3}$ ). We refer to [9] for more details on these and related issues. Studying (non-local) Kolmogorov equations associated to (1) in its full generality remains nonetheless a very interesting problem.

Denoting by $u(\cdot, x)$ the solution to the stochastic equation (1), we have proved in the previous section that the semigroup

$$
P_{t} f(x):=\mathbb{E} f(u(t, x)), \quad f \in B_{b}(H)
$$

admits a (not necessarily unique) invariant measure $\nu$. As is well-known, $P_{t}$ can be extended to a strongly continuous Markovian semigroup of contractions on $L_{p}(H, \nu), p \geq 1$. In the following we shall denote the extension of $P_{t}$ to $L_{p}(H, \nu)$ again by $P_{t}$.

Let us define the operator $(L, D(L))$ in $L_{1}(H, \nu)$ by

$$
\begin{aligned}
& L f(x)=-\langle A x, D f(x)\rangle+\mathcal{I} f(x), \quad x \in V, \\
& \mathcal{I} f(x)=\int_{Z}[f(x+G(z))-f(x)-\langle D f(x), G(z)\rangle] m(d z), \\
& D(L)=\left\{f \in C_{b}^{1,1}(H) \cap C_{b}^{1}\left(V^{\prime}\right)\right\},
\end{aligned}
$$

where $C_{b}^{1,1}(H)$ denotes the set of functions $\phi \in C_{b}^{1}(H)$ with $D \phi: H \rightarrow H$ Lipschitz continuous. Note that the nonlocal term $\mathcal{I} f$ in the definition of $L$ is a well-defined element of $L_{1}(H, \nu)$ for $f \in C_{b}^{1,1}(H)$. In fact, the fundamental theorem of calculus yields

$$
\begin{aligned}
& |f(x+G(z))-f(x)-\langle D f(x), G(z)\rangle| \\
& \quad \leq\left|\int_{0}^{1}\langle D f(x+\theta G(z)), G(z)\rangle d \theta-\langle D f(x), G(z)\rangle\right| \leq|D f|_{\dot{C}^{0,1}(H \rightarrow H)}|G(z)|^{2}
\end{aligned}
$$

therefore, since $G \in L_{2}(Z, m)$, we have that $|\mathcal{I} f| \lesssim 1$, thus also $\mathcal{I} f \in L_{1}(H, \nu)$. Similarly, $x \mapsto\langle A x, D f(x)\rangle \in L_{1}(H, \nu)$, as explained below in a slightly more general setting.

By a computation based on Itô's formula one can see that the infinitesimal generator of $P_{t}$ in $L_{1}(H, \nu)$ acts on smooth enough functions as the operator $L$ just defined. Since $P_{t}$ is a contraction for all $t \geq 0$, we have that $(L, D(L))$ is dissipative in $L_{1}(H, \nu)$. The question of $L_{1}$-uniqueness then arises naturally: is $P_{t}$ the only strongly continuous semigroup on $L_{1}(H, \nu)$ such that its infinitesimal generator extends $(L, D(L))$ ? Under a "regularizability" hypothesis on $A$, we shall give an affirmative answer to this question, proving that the closure of $L$ in $L_{1}(H, \nu)$ generates a strongly continuous semigroup. In fact, since $L$ is dissipative, this will imply that the semigroup coincides with $P_{t}$. 
Throughout this section we shall assume that there exists a sequence of monotone operators $A^{\varepsilon} \in \dot{C}^{0,1}(H \rightarrow H) \cap C_{b}^{1}\left(V \rightarrow V^{\prime}\right)$ such that $A^{\varepsilon} x \rightarrow A x$ in $V^{\prime}$ for all $x \in V$ and $\left|A^{\varepsilon} x\right|_{V^{\prime}} \leq N\left(|x|_{V}^{p-1}+1\right)$ with $N$ independent of $\varepsilon$.

We are going to prove that $L$ is dissipative in $L_{1}(H, \nu)$ just assuming that $\nu$ is an infinitesimally invariant for $L$ satisfying the integrability condition

$$
x \mapsto|x|_{V}^{p}+|x|_{H} \in L_{1}(H, \nu) .
$$

More precisely, the assumption of $\nu$ being infinitesimally invariant amounts to assuming that

$$
\int_{H} L f d \nu=0 \quad \forall f \in D(L)
$$

Note that (19) and $f \in D(L)$ imply that $L f \in L_{1}(H, \nu)$, so that the above condition is meaningful. In fact, one has $\mathcal{I} f \in L_{1}(H, \nu)$ for all $f \in C_{b}^{1,1}(H)$, as seen above, and

$$
|\langle A x, D f(x)\rangle| \leq|A x|_{V^{\prime}} \sup _{y \in V}|D f(y)| \lesssim|x|_{V}^{p}+1 \in L_{1}(H, \nu) .
$$

Let us recall that any invariant measure is infinitesimally invariant, but the converse does not hold, in general. Moreover, any invariant measure for (1) satisfies the integrability condition (19) thanks to Theorem 2.6.

Lemma 3.1. The operator $(L, D(L))$ is dissipative, hence closable, in $L_{1}(H, \nu)$.

Proof. Let $f \in D(L)$ and $\gamma_{\varepsilon} \in C^{2}(\mathbb{R})$ be a convex function such that $\gamma_{\varepsilon}^{\prime} \in$ $C_{b}^{1}(\mathbb{R})$ is a smooth approximation of the signum graph

$$
\operatorname{sgn}(x)= \begin{cases}-1, & x<0 \\ {[-1,1],} & x=0 \\ 1, & x>0\end{cases}
$$

Then we have

$$
L \gamma_{\varepsilon}(f)=\langle A x, D f\rangle \gamma_{\varepsilon}^{\prime}(f)+\mathcal{I} \gamma_{\varepsilon}(f),
$$

where, by a direct calculation,

$$
\begin{aligned}
\mathcal{I} \gamma_{\varepsilon}(f) & -\gamma_{\varepsilon}^{\prime}(f) \mathcal{I} f \\
= & \int_{Z}\left[\gamma_{\varepsilon}(f(x+G(z)))-\gamma_{\varepsilon}(f(x))-\gamma_{\varepsilon}^{\prime}(f(x))(f(x+G(z))-f(x))\right] m(d z) \\
= & : R_{\varepsilon}(f) .
\end{aligned}
$$

Since $\gamma_{\varepsilon}$ is convex and differentiable, we infer that $R_{\varepsilon}(f) \geq 0$. Therefore, taking the previous inequality into account and the infinitesimal invariance of $\nu$, one has, integrating (20) with respect to $\nu$,

$$
\int L \gamma_{\varepsilon}(f) d \nu=0=\int \gamma_{\varepsilon}^{\prime}(f) L f d \nu+\int R_{\varepsilon}(f) d \nu
$$

hence $\int \gamma_{\varepsilon}^{\prime}(f) L f d \nu \leq 0$, and passing to the limit as $\varepsilon \rightarrow 0$,

$$
\int L f \xi d \nu \leq 0
$$

where $\xi \in L_{\infty}(H, \nu), \xi \in \operatorname{sgn}(f) \nu$-a.e. Since $L_{1}(H, \nu)^{\prime}=L_{\infty}(H, \nu)$, recalling that the duality map $J: L_{1}(H, \nu) \rightarrow 2^{L_{\infty}(H, \nu)}$ is given by

$$
J: u \mapsto\left\{v \in L_{\infty}(H, \nu): v \in|u|_{L_{1}(H, \nu)} \operatorname{sgn}(u) \quad \nu \text {-a.e. }\right\}
$$


(see e.g. $[4, \S 1.1]$ ), we infer by the previous inequality that $L$ is dissipative in $L_{1}(H, \nu)$.

The following result gives a positive answer to the $L_{1}$-uniqueness question posed above.

THEOREM 3.2. Let $(\bar{L}, D(\bar{L}))$ be the closure of the Kolmogorov operator $L$ in $L_{1}(H, \nu)$. Then $(\bar{L}, D(\bar{L}))$ generates a strongly continuous Markovian semigroup of contractions $T_{t}$ in $L_{1}(H, \nu)$, for which $\nu$ is an invariant measure.

Proof. By the Lumer-Phillips theorem (see e.g. [22, §1.4, Thm. 4.3]), $\bar{L}$ generates a strongly continuous semigroup of contractions if $R(\alpha I-\bar{L})$ is dense in $L_{1}(H, \nu)$ for some $\alpha>0$.

Consider the regularized equation

$$
d u(t)+A^{\varepsilon \lambda} u d t=\int_{Z} G(z) d \bar{\mu}(d t, d z), \quad u(0)=x \in H,
$$

with

$$
A^{\varepsilon \lambda} x:=\int_{H} e^{\lambda C} A^{\varepsilon}\left(e^{\lambda C} x+y\right) \Upsilon_{\frac{1}{2} C^{-1}\left(e^{2 \lambda C}-1\right)}(d y), \quad \lambda>0,
$$

where $C: D(C) \subset V \rightarrow H$ is a self-adjoint, negative definite linear operator such that $C^{-1}$ is of trace class, and $\Upsilon_{Q}$ stands for a centered Gaussian measure on $H$ with covariance operator $Q$. Then, by the Cameron-Martin formula (see e.g. [8, p. 10]), one has

$$
A^{\varepsilon \lambda} \in C^{\infty}(H \rightarrow H), \quad\left(A^{\varepsilon \lambda}\right)^{\prime} \in C_{b}^{\infty}(H \rightarrow \mathcal{L}(H \rightarrow H))
$$

and $A^{\varepsilon \lambda} x \rightarrow A^{\varepsilon} x$ for all $x \in H$ as $\lambda \rightarrow 0$ (see e.g. [8, $\left.\S 2.3-2.4\right]$ for details). Moreover, $A^{\varepsilon \lambda}$ inherits the monotonicity of $A^{\varepsilon}$, and

$$
\left(A^{\varepsilon \lambda}\right)^{\prime} x=\int_{H} e^{\lambda C}\left(A^{\varepsilon}\right)^{\prime}\left(e^{\lambda C} x+y\right) e^{\lambda C} \Upsilon_{\frac{1}{2} C^{-1}\left(e^{2 \lambda C}-1\right)}(d y),
$$

so that $A^{\varepsilon \lambda} \in C_{b}^{1}\left(V \rightarrow V^{\prime}\right)$.

Since $A^{\varepsilon \lambda}$ is Lipschitz continuous on $H,(21)$ admits a unique strong solution $u_{\varepsilon \lambda}$ (e.g. by [19, thm. 34.7]). Set

$$
f_{\varepsilon \lambda}(x):=\mathbb{E} \int_{0}^{\infty} e^{-\alpha t} \varphi\left(u_{\varepsilon \lambda}(t, x)\right) d t, \quad x \in H,
$$

where $\varphi \in D(L)$ and $\alpha>0$ are fixed. Since $A^{\varepsilon \lambda} \in C^{1}(H \rightarrow H)$, one has, thanks to $\left[\mathbf{1 9}\right.$, thm. 36.9], that $x \mapsto u_{\varepsilon \lambda}(t, x)$ is Fréchet differentiable for all $t \geq 0$, and its Fréchet derivative acting on an arbitrary $y \in H$, denoted by $v_{\varepsilon \lambda}^{y}:=D u_{\varepsilon \lambda}[y]$, solves the initial value problem (in the $\mathbb{P}$-a.s. sense)

$$
\frac{d}{d t} v_{\varepsilon \lambda}^{y}+\left(A^{\varepsilon \lambda}\right)^{\prime}\left(u_{\varepsilon \lambda}\right) v_{\varepsilon \lambda}^{y}=0, \quad v_{\varepsilon \lambda}^{y}(0, x)=y .
$$

A computation based on Itô's lemma for the square of the norm and the monotonicity of $A^{\varepsilon \lambda}$ reveals that $x \mapsto u_{\varepsilon \lambda}(\cdot, x) \in \dot{C}^{0,1}\left(H \rightarrow \mathbb{H}_{2}(T)\right)$ for all $T \geq 0$, and

$$
\left|x \mapsto u_{\varepsilon \lambda}(t, x)\right|_{\dot{C}^{0,1}(H \rightarrow H)} \leq 1 \quad \forall t \geq 0 .
$$

This immediately implies that $\left|v_{\varepsilon \lambda}^{y}\right| \leq|y|$ for all $y \in H$, as the operator norm of the Fréchet derivative of a Lipschitz continuous function cannot exceed its Lipschitz constant. Moreover, since $\left(A^{\varepsilon \lambda}\right)^{\prime}(\xi) \in C_{b}^{0}(H \rightarrow H)$ for all $\xi \in H$, from (23) we infer that $x \mapsto u_{\varepsilon \lambda}(t, x)$ is continuously differentiable $\mathbb{P}$-a.s. for all $t \geq 0$ (e.g. by 
[11, §X.8]). Applying the chain rule for Fréchet derivatives (see e.g. [2, Prop. 1.4]) in (22), taking into account that $\varphi \in C_{b}^{1,1}(H)$ and $u_{\varepsilon \lambda}$ is Fréchet differentiable with $\left|D u_{\varepsilon \lambda}(t)\right|$ bounded uniformly over $t$, we get

$$
D f_{\varepsilon \lambda}(x)[y]=\mathbb{E} \int_{0}^{\infty} e^{-\alpha t} D \varphi\left(u_{\varepsilon \lambda}(t, x)\right) v_{\varepsilon \lambda}^{y}(t, x) d t
$$

for all $y \in H$, which also immediately yields

$$
\left|D f_{\varepsilon \lambda}(x)[y]\right| \lesssim|y| \quad \forall y \in H
$$

that is $f_{\varepsilon \lambda} \in C_{b}^{1}(H)$. In order to conclude that $f_{\varepsilon \lambda} \in C_{b}^{1,1}(H)$ we thus have to prove that $D f_{\varepsilon \lambda} \in \dot{C}^{0,1}(H \rightarrow H)$. Let us observe that we can write

$$
\begin{aligned}
\mid D f_{\varepsilon \lambda}(x) & {[y]-D f_{\varepsilon \lambda}(x)[z] \mid } \\
\leq & \mathbb{E} \int_{0}^{\infty} e^{-\alpha t}\left|D \varphi\left(u_{\varepsilon \lambda}(t, x)\right) v_{\varepsilon \lambda}^{y}(t, x)-D \varphi\left(u_{\varepsilon \lambda}(t, z)\right) v_{\varepsilon \lambda}^{y}(t, z)\right| d t \\
\leq & \mathbb{E} \int_{0}^{\infty} e^{-\alpha t}\left|D \varphi\left(u_{\varepsilon \lambda}(t, x)\right) v_{\varepsilon \lambda}^{y}(t, x)-D \varphi\left(u_{\varepsilon \lambda}(t, x)\right) v_{\varepsilon \lambda}^{y}(t, z)\right| d t \\
& +\mathbb{E} \int_{0}^{\infty} e^{-\alpha t}\left|D \varphi\left(u_{\varepsilon \lambda}(t, x)\right) v_{\varepsilon \lambda}^{y}(t, z)-D \varphi\left(u_{\varepsilon \lambda}(t, z)\right) v_{\varepsilon \lambda}^{y}(t, z)\right| d t,
\end{aligned}
$$

where, recalling that $x \mapsto u_{\varepsilon \lambda}(t, x)$ and $v_{\varepsilon \lambda}(t)$ are respectively Lipschitz and bounded uniformly over $\varepsilon, \lambda$ and $t$, and that $\varphi \in C_{b}^{1,1}(H)$,

$$
\begin{aligned}
& \left|D \varphi\left(u_{\varepsilon \lambda}(t, x)\right) v_{\varepsilon \lambda}^{y}(t, z)-D \varphi\left(u_{\varepsilon \lambda}(t, z)\right) v_{\varepsilon \lambda}^{y}(t, z)\right| \\
& \leq|D \varphi|_{\dot{C}^{0,1}(H \rightarrow H)}\left|u_{\varepsilon \lambda}(t, x)-u_{\varepsilon \lambda}(t, z)\right|\left|v_{\varepsilon \lambda}^{y}(t, z)\right| \\
& \lesssim|x-z||y| .
\end{aligned}
$$

Moreover, we also have

$\left|D \varphi\left(u_{\varepsilon \lambda}(t, x)\right) v_{\varepsilon \lambda}^{y}(t, x)-D \varphi\left(u_{\varepsilon \lambda}(t, x)\right) v_{\varepsilon \lambda}^{y}(t, z)\right| \leq|D \varphi|_{C^{0}(H \rightarrow H)}\left|v_{\varepsilon \lambda}^{y}(t, x)-v_{\varepsilon \lambda}^{y}(t, z)\right|$,

from which it follows that in order to show that $D f_{\varepsilon \lambda}$ is Lipschitz on $H$ it suffices to prove that $x \mapsto v_{\varepsilon \lambda}(t, x)$ is Lipschitz on $H$. We have

$\frac{d}{d t}\left(v_{\varepsilon \lambda}^{y}(t, x)-v_{\varepsilon \lambda}^{y}(t, z)\right)+\left(A^{\varepsilon \lambda}\right)^{\prime}\left(u_{\varepsilon \lambda}(t, x)\right) v_{\varepsilon \lambda}^{y}(t, x)-\left(A^{\varepsilon \lambda}\right)^{\prime}\left(u_{\varepsilon \lambda}(t, z)\right) v_{\varepsilon \lambda}^{y}(t, z)=0$,

hence, taking scalar products with $v_{\varepsilon \lambda}^{y}(t, x)-v_{\varepsilon \lambda}^{y}(t, z)$,

$$
\frac{1}{2} \frac{d}{d t}\left|v_{\varepsilon \lambda}^{y}(t, x)-v_{\varepsilon \lambda}^{y}(t, z)\right|^{2}+I=0,
$$

where $I \equiv I(\varepsilon, \lambda, t, x, z, y)$ satisfies

$$
\begin{aligned}
I= & \left\langle\left(A^{\varepsilon \lambda}\right)^{\prime}\left(u_{\varepsilon \lambda}(t, x)\right)\left(v_{\varepsilon \lambda}^{y}(t, x)-v_{\varepsilon \lambda}^{y}(t, z)\right), v_{\varepsilon \lambda}^{y}(t, x)-v_{\varepsilon \lambda}^{y}(t, z)\right\rangle \\
& +\left\langle\left(A^{\varepsilon \lambda}\right)^{\prime}\left(u_{\varepsilon \lambda}(t, x)\right) v_{\varepsilon \lambda}^{y}(t, z)-\left(A^{\varepsilon \lambda}\right)^{\prime}\left(u_{\varepsilon \lambda}(t, z)\right) v_{\varepsilon \lambda}^{y}(t, z), v_{\varepsilon \lambda}^{y}(t, x)-v_{\varepsilon \lambda}^{y}(t, z)\right\rangle \\
\geq & \left\langle\left(A^{\varepsilon \lambda}\right)^{\prime}\left(u_{\varepsilon \lambda}(t, x)\right) v_{\varepsilon \lambda}^{y}(t, z)-\left(A^{\varepsilon \lambda}\right)^{\prime}\left(u_{\varepsilon \lambda}(t, z)\right) v_{\varepsilon \lambda}^{y}(t, z), v_{\varepsilon \lambda}^{y}(t, x)-v_{\varepsilon \lambda}^{y}(t, z)\right\rangle,
\end{aligned}
$$

once one takes into account that $\left(A^{\varepsilon \lambda}\right)^{\prime}\left(u_{\varepsilon \lambda}(t, x)\right)$ is a positive linear operator, because $A^{\varepsilon \lambda}: H \rightarrow H$ is monotone and differentiable. Then we also get, recalling 
that $\left|v_{\varepsilon \lambda}^{y}(t, z)\right| \leq|y|$

$$
\begin{aligned}
-I \leq & \frac{1}{2}\left|\left(\left(A^{\varepsilon \lambda}\right)^{\prime}\left(u_{\varepsilon \lambda}(t, x)\right)-\left(A^{\varepsilon \lambda}\right)^{\prime}\left(u_{\varepsilon \lambda}(t, z)\right)\right) v_{\varepsilon \lambda}^{y}(t, z)\right|^{2} \\
& +\frac{1}{2}\left|v_{\varepsilon \lambda}^{y}(t, x)-v_{\varepsilon \lambda}^{y}(t, z)\right|^{2} \\
\leq & \frac{1}{2}|y|^{2}\left[\left(A^{\varepsilon \lambda}\right)^{\prime}\right]_{1}^{2}\left|u_{\varepsilon \lambda}(t, x)-u_{\varepsilon \lambda}(t, z)\right|^{2}+\frac{1}{2}\left|v_{\varepsilon \lambda}^{y}(t, x)-v_{\varepsilon \lambda}^{y}(t, z)\right|^{2} \\
& \lesssim|y|^{2}|x-z|^{2}+\left|v_{\varepsilon \lambda}^{y}(t, x)-v_{\varepsilon \lambda}^{y}(t, z)\right|^{2} .
\end{aligned}
$$

In the last step we have used that $\left(A^{\varepsilon \lambda}\right)^{\prime} \in C_{b}^{\infty}(H \rightarrow \mathcal{L}(H \rightarrow H)$ and that $x \mapsto$ $u_{\varepsilon \lambda}(t, x)$ is Lipschitz. Gronwall's inequality then yields

$$
\left|v_{\varepsilon \lambda}(t, x)-v_{\varepsilon \lambda}(t, z)\right| \lesssim|x-z|,
$$

thus concluding the proof that $f_{\varepsilon \lambda} \in C_{b}^{1,1}(H)$.

Let us now prove that $f_{\varepsilon \lambda} \in C_{b}^{1}\left(V^{\prime}\right)$ : in view of (24), it is enough to prove that $\left|v_{\varepsilon \lambda}^{y}(x)\right|_{V^{\prime}} \leq|y|_{V^{\prime}}$. Here we regard $\varphi$ as a function from $V^{\prime}$ to $\mathbb{R}$ and $x \mapsto u_{\varepsilon \lambda}(t, x)$ as a map from $V^{\prime}$ to itself, so that $v_{\varepsilon \lambda}(t, x) \in \mathcal{L}\left(V^{\prime} \rightarrow V^{\prime}\right)$ and $v_{\varepsilon \lambda}^{y}(t, x) \in V^{\prime}$. Let $J: V^{\prime} \rightarrow V^{\prime \prime} \simeq V$ denote the duality map between $V^{\prime}$ and $V$ (or equivalently, let $J=F^{-1}$, with $F$ the duality map between $V$ and $V^{\prime}$ ). Multiplying both sides of (23) by $J\left(v_{\varepsilon \lambda}^{y}(t, x)\right)$, in the sense of the duality pairing between $V^{\prime}$ and $V$, we obtain, taking into account that $\left(A^{\varepsilon \lambda}\right)^{\prime}$ is positive, $\left|v_{\varepsilon \lambda}^{y}(x)\right|_{V^{\prime}} \leq|y|_{V^{\prime}}$. We have thus proved that $f_{\varepsilon \lambda} \in D(L)$. This in turn implies that $f_{\varepsilon \lambda}$ satisfies

$$
\begin{aligned}
& \alpha f_{\varepsilon \lambda}(x)+V_{V^{\prime}}\left\langle A^{\varepsilon \lambda} x, D f_{\varepsilon \lambda}(x)\right\rangle_{V} \\
& \quad-\int_{Z}\left[f_{\varepsilon \lambda}(x+G(z))-f_{\varepsilon \lambda}(x)-\left\langle D f_{\varepsilon \lambda}(x), G(z)\right\rangle\right] m(d z)=\varphi(x), \quad x \in H,
\end{aligned}
$$

hence also

$$
\alpha f_{\varepsilon \lambda}(x)+\left\langle A x, D f_{\varepsilon \lambda}(x)\right\rangle-\mathcal{I} f_{\varepsilon \lambda}(x)=\varphi(x)+\left\langle A x-A^{\varepsilon \lambda} x, D f_{\varepsilon \lambda}(x)\right\rangle,
$$

and

$$
\left|\alpha f_{\varepsilon \lambda}+\left\langle A x, D f_{\varepsilon \lambda}\right\rangle-\mathcal{I} f_{\varepsilon \lambda}\right|_{L_{1}(H, \nu)} \leq|\varphi|_{L_{1}(H, \nu)}+\left|\left\langle A x-A^{\varepsilon \lambda} x, D f_{\varepsilon \lambda}\right\rangle\right|_{L_{1}(H, \nu)} .
$$

Note that $\left|D f_{\varepsilon \lambda}(x)\right|_{V} \lesssim 1$ thanks to the above bound on $\left|v_{\varepsilon \lambda}(x)\right|_{V^{\prime}}$, so that

$$
\begin{aligned}
\int_{H} \mid\langle A x & \left.-A^{\varepsilon \lambda} x, D f_{\varepsilon \lambda}(x)\right\rangle \mid \nu(d x) \\
& \lesssim \int_{H}\left|A x-A^{\varepsilon} x\right|_{V^{\prime}} \nu(d x)+\int_{H}\left|A^{\varepsilon} x-A^{\varepsilon \lambda} x\right|_{V^{\prime}} \nu(d x)
\end{aligned}
$$

which converges to 0 as $\lambda \rightarrow 0$ and $\varepsilon \rightarrow 0$ by the dominated convergence theorem. In fact, thanks to the hypotheses on $A$ and $A^{\varepsilon}$, we have $\left|A x-A^{\varepsilon} x\right|_{V^{\prime}} \lesssim|x|_{V}^{p}+1$ for all $x \in V$, and $\nu$ is concentrated on $V$ by (19). Moreover, since $H \hookrightarrow V^{\prime}$ is continuous and $\left|A^{\varepsilon \lambda} x\right| \leq\left|A^{\varepsilon} x\right|$ for all $x \in H$, we have $\left.\left|A^{\varepsilon} x-A^{\varepsilon \lambda} x\right|_{V^{\prime}}|\lesssim| x\right|_{H}+1 \in L_{1}(H, \nu)$, because of $(19)$. We have thus shown that

$$
\lim _{\varepsilon \rightarrow 0} \lim _{\lambda \rightarrow 0}\left(\alpha f_{\varepsilon \lambda}+\left\langle A x, D f_{\varepsilon \lambda}\right\rangle-\mathcal{I} f_{\varepsilon \lambda}\right)=\varphi
$$

in $L_{1}(H, \nu)$, i.e. that $R(\alpha I-L)$ is dense in $L_{1}(H, \nu)$, because $D(L)$ is dense in $L_{1}(H, \nu)$. Since $L$ is also dissipative, we immediately infer that $\bar{L}$ is $m$-dissipative in $L_{1}(H, \nu)$. 
Let us denote the strongly continuous semigroup of contractions on $L_{1}(H, \nu)$ with generator $\bar{L}$ by $T_{t}$. Let us now prove that $T_{t}$ is Markovian: for this it is enough to show that

$$
\int_{H} \bar{L} f 1_{\{f>1\}} d \nu \leq 0 \quad \forall f \in D(\bar{L})
$$

(see e.g. [26, p. 109]). Let $\gamma_{\varepsilon} \in C^{2}(\mathbb{R})$ be a convex function such that $\gamma_{\varepsilon}^{\prime} \in C_{b}^{1}(\mathbb{R})$ is a smooth approximation of $x \mapsto 1_{1,+\infty}(x)$. Then, proceeding as in the proof of the previous lemma, we obtain the claim for all $f \in D(L)$ first, and for all $f \in D(\bar{L})$ by density.

In order to prove that $\nu$ is an invariant measure for $T_{t}$, let us observe that one has, by definition of infinitesimal invariance and by a density argument,

$$
\int_{H} \bar{L} f d \nu=0 \quad \forall f \in D(\bar{L}) .
$$

Since $T_{t} f \in D(\bar{L})$ for all $t \geq 0$ if $f \in D(\bar{L})$, we have, by the infinitesimal invariance of $\nu$,

$$
\int_{H} T_{t} f d \nu=\int_{H} f d \nu+\int_{0}^{t} \int_{H} \bar{L} T_{s} f d \nu d s=\int_{H} f d \nu
$$

for all $f \in D(\bar{L})$, thus also for all $f \in L_{1}(H, \nu)$ by density.

REMARK 3.3. The theorem implies that if $\nu$ is an invariant measure to the stochastic equation (1) satisfying the integrability condition (19), then for all $f \in$ $B_{b}(H)$, one has that $T_{t} f$ is a $\nu$-version of $P_{t} f$ for all $t \geq 0$.

REMARK 3.4. The dissipativity of $L$ in $L_{2}(H, \nu)$ is easier to prove: in fact, for $f \in D(L)$, we have

$$
L\left(f^{2}\right)=2 f L f+\Gamma(f, f)
$$

where

$$
\Gamma(f, f)=\int_{Z}|f(x+G(z))-f(x)|^{2} m(d z) \geq 0
$$

is the so-called carré du champ operator associated to $\mathcal{I}$, which is defined as

$$
\Gamma(f, g):=\mathcal{I}(f g)-f \mathcal{I} g-g \mathcal{I} f
$$

and takes the form

$$
\Gamma(f, g)=\int_{Z}(f(x+G(z))-f(x))(g(x+G(z))-g(x)) m(d z) .
$$

In particular one has the integration by parts formula

$$
\int f L f d \nu=-\frac{1}{2} \int \Gamma(f, f) d \nu
$$

However, as one might expect, one needs stronger integrability assumptions on $\nu$ to prove the essential $m$-dissipativity of $L$, e.g. (roughly) of the type $x \mapsto|A x|^{2} \in$ $L_{1}(H, \nu)$. Such an assumption would in turn require the data of the problem to be much more regular. 


\section{Applications}

4.1. SDEs with monotone drift. If $V=H=\mathbb{R}^{d}$, so that (1) reduces to an ordinary stochastic differential equation with monotone drift, our results on ergodicity can be recovered applying [13, Thm. 2], which provides existence and uniqueness of strong solutions (even in a more general situation than that treated here), and [25, Thm. I.25], which establishes boundedness in probability for the solution by a Lyapunov-type criterion. In our case one can choose as Lyapunov function simply $V(x)=|x|^{2}$.

4.2. Stochastic equations with drift in divergence form. Let $D \subset \mathbb{R}^{d}$ be a bounded domain with smooth boundary, and set $H:=L_{2}(D), V=W_{p}^{1}(D)$, $V^{\prime}=W_{q}^{-1}(D)$, with $p>2, p^{-1}+q^{-1}=1$. Here $\stackrel{\circ}{W}_{p}^{1}(D), p \in[1, \infty[$, stands for the closure of $C_{c}^{\infty}(D)$, the set of real-valued functions on $D$ with compact support, with respect to the norm

$$
|\phi|_{W_{p}^{1}(D)}:=|\phi|_{L_{p}(D)}+|\nabla \phi|_{L_{p}(D)}
$$

(see e.g. [16, $\S 8.0]$ ). Note that $V \hookrightarrow H$ is compact by a Sobolev embedding theorem (see e.g. [2, Thm. 0.4]). Consider the operator $A: V \rightarrow V^{\prime}$ defined by

$$
A u:=-\operatorname{div}(a(\nabla u)),
$$

which must be interpreted, as usual, as

$$
\langle A u, v\rangle=\int_{D}\langle a(\nabla u(\xi)), \nabla v(\xi)\rangle_{\mathbb{R}^{d}} d \xi \quad \forall v \in V .
$$

Here $a \in C^{0}\left(\mathbb{R}^{d} \rightarrow \mathbb{R}^{d}\right)$ is a monotone function satisfying the polynomial growth condition $|a(x)| \lesssim|x|^{p-1}+1$ and the coercivity condition $x a(x) \gtrsim|x|^{p}-1$. Note that the $p$-Laplacian is a special case of this example obtained taking $a(x)=|x|^{p-2} x$ (see e.g. $[\mathbf{2 4}, \S 4.1]$ for a related example).

Let $\tilde{a}_{\varepsilon} \in \dot{C}^{0,1}\left(\mathbb{R}^{d} \rightarrow \mathbb{R}^{d}\right), \tilde{a}_{\varepsilon}(x):=\varepsilon^{-1}\left(x-(I+\varepsilon a)^{-1} x\right)$ be the Yosida approximation of $a$, so that, in particular $\tilde{a}_{\varepsilon}$ is monotone and Lipschitz continuous for each $\varepsilon>0$ with Lipschitz constant $2 / \varepsilon$ (see e.g. $[4, \S 2.1]$ ). Set $a_{\varepsilon}=\tilde{a}_{\varepsilon} * \zeta_{\varepsilon}$, where $\left\{\zeta_{\varepsilon}\right\}$ is a standard sequence of mollifiers (in particular $a_{\varepsilon} \in C^{\infty}, a_{\varepsilon}^{\prime} \in C_{b}^{\infty}$ ), and define the operator $A^{\varepsilon}$ on smooth functions as

$$
A^{\varepsilon} u=-(I-\varepsilon \Delta)^{-1} \operatorname{div}\left(a_{\varepsilon}\left(\nabla(I-\varepsilon \Delta)^{-1} u\right)\right),
$$

where $\Delta$ stands for the Dirichlet Laplacian on $D$. We are going to show that $A^{\varepsilon}$ satisfies the assumptions of the previous section. For this we shall need some elliptic regularity results, which we recall here (see e.g. [16, $\S 8.5]$ for details).

Lemma 4.1. Let $f \in L_{p}(D), p \geq 2$. Then there exists $\varepsilon_{1}$ such that, for all $\varepsilon<\varepsilon_{1}$, there exists a unique solution $u \in \stackrel{\circ}{W}_{p}^{1}$ to the equation

$$
u-\varepsilon \Delta u=f
$$

on D with Dirichlet boundary conditions. Moreover u satisfies the estimate

$$
|u|_{L_{p}(D)}+\varepsilon^{1 / 2}|u|_{W_{p}^{1}(D)} \leq N|f|_{L_{p}(D)},
$$

where $N$ does not depend on $\varepsilon$. 
Let us first show that $A^{\varepsilon}$ is well-defined both as an operator from $H$ to itself, as well as from $V$ to $V^{\prime}$. Using the notation

$$
v^{(\varepsilon)}=(I-\varepsilon \Delta)^{-1} v,
$$

we may write

$$
\left\langle A^{\varepsilon} u, v\right\rangle=\int_{D}\left\langle a_{\varepsilon}\left(\nabla u^{(\varepsilon)}\right), \nabla v^{(\varepsilon)}\right\rangle_{\mathbb{R}^{d}} d \xi
$$

Note that if $v \in H$, then $v^{(\varepsilon)} \in \stackrel{\circ}{W}_{2}^{1}$ and

$$
\left|\nabla v^{(\varepsilon)}\right|_{H} \leq\left|v^{(\varepsilon)}\right|_{W_{2}^{1}(D)} \lesssim \varepsilon|v|_{H}
$$

Moreover, since $a_{\varepsilon}$ is Lipschitz continuous, we have

$$
\left|a_{\varepsilon}\left(\nabla u^{(\varepsilon)}\right)\right| \leq\left|a_{\varepsilon}\left(\nabla u^{(\varepsilon)}\right)-a_{\varepsilon}(0)\right|+\left|a_{\varepsilon}(0)\right| \lesssim \varepsilon\left|\nabla u^{(\varepsilon)}\right|_{H}+\left|a_{\varepsilon}(0)\right|,
$$

thus also

$$
\left|\left\langle A^{\varepsilon} u, v\right\rangle\right| \leq\left|a_{\varepsilon}\left(\nabla u^{(\varepsilon)}\right)\right|_{H}\left|\nabla v^{(\varepsilon)}\right|_{H} \lesssim \varepsilon\left(|u|_{H}+a_{\varepsilon}(0)\right)|v|_{H}
$$

which shows that $A^{\varepsilon}$ is well-defined from $H$ to itself. Similarly, if $u, v \in V=$ $W_{p}^{1}(D)$, we have, by Hölder's inequality,

$$
\left|\left\langle A^{\varepsilon} u, v\right\rangle\right| \leq\left|a_{\varepsilon}\left(\nabla u^{(\varepsilon)}\right)\right|_{L_{q}(D)}\left|\nabla v^{(\varepsilon)}\right|_{L_{p}(D)} \lesssim\left(|u|_{V}+1\right)|v|_{V},
$$

where we have used again Lemma 4.1 and $\|\cdot\|_{L_{q}(D)} \lesssim\|\cdot\|_{L_{p}(D)}$ for $p>q$ and $D$ bounded. We have thus shown that $A^{\varepsilon}$ is well-defined from $V$ to $V^{\prime}$.

The monotonicity of $A^{\varepsilon}$, both as an operator from $H$ to itself and from $V$ to $V^{\prime}$ is immediate by (26) and the monotonicity of $a_{\varepsilon}$.

Let us now show that $A^{\varepsilon}$ is Lipschitz continuous on $H$. In fact, taking into account Lemma 4.1, we have

$$
\begin{aligned}
\left|\left\langle A^{\varepsilon} u-A^{\varepsilon} v, w\right\rangle\right| & =\left|\left\langle a_{\varepsilon}\left(\nabla u^{(\varepsilon)}\right)-a_{\varepsilon}\left(\nabla v^{(\varepsilon)}\right), \nabla w^{(\varepsilon)}\right\rangle\right| \\
& \lesssim_{\varepsilon}\left|\nabla\left(v^{(\varepsilon)}-w^{(\varepsilon)}\right)\right|\left|\nabla w^{(\varepsilon)}\right| \lesssim_{\varepsilon}|u-v|_{H}|w|_{H} .
\end{aligned}
$$

Since $a_{\varepsilon} \in C^{1}$, a direct computation yields that $A^{\varepsilon}$ is Gâteaux differentiable from $V$ to $V^{\prime}$ with Gâteaux differential

$$
\left\langle\left(A^{\varepsilon}\right)^{\prime}(u)[v], w\right\rangle=\int_{D}\left\langle a_{\varepsilon}^{\prime}\left(\nabla u^{(\varepsilon)}\right) \nabla v^{(\varepsilon)}, \nabla w^{(\varepsilon)}\right\rangle_{\mathbb{R}^{d}} d \xi
$$

for all $u, v, w \in V$. Note that the integral is well defined because $\left|a_{\varepsilon}^{\prime}(x)\right| \lesssim 1$ for all $x \in \mathbb{R}^{d}$, since $a_{\varepsilon}$ is Lipschitz continuous. By a well-known criterion, we can conclude that $A^{\varepsilon} \in C^{1}\left(V \rightarrow V^{\prime}\right)$ if we show that $\left(A^{\varepsilon}\right)^{\prime}$ in $(27)$ is continuous as a map $V \rightarrow \mathcal{L}\left(V \rightarrow V^{\prime}\right)$. Let $u_{n} \rightarrow u$ in $\stackrel{\circ}{W}_{p}^{1}(D)$ as $n \rightarrow \infty$ : applying Hölder's inequality and Lemma 4.1 repeatedly, we obtain

$$
\begin{aligned}
\sup _{|v|_{V} \leq 1} \sup _{|w|_{V}} \leq 1 & \left\langle\left(A^{\varepsilon}\right)^{\prime}\left(u_{n}\right)[v]-\left(A^{\varepsilon}\right)^{\prime}(u)[v], w\right\rangle \\
& \leq\left|\nabla w^{(\varepsilon)}\right|_{L_{p}(D)}\left|\left(a_{\varepsilon}^{\prime}\left(\nabla u_{n}^{(\varepsilon)}\right)-a_{\varepsilon}^{\prime}\left(\nabla u^{(\varepsilon)}\right)\right) \nabla v^{(\varepsilon)}\right|_{L_{p /(p-1)}(D)} \\
& \lesssim\left|\nabla v^{(\varepsilon)}\right|_{L_{p}(D)}\left|a_{\varepsilon}^{\prime}\left(\nabla u_{n}^{(\varepsilon)}\right)-a_{\varepsilon}^{\prime}\left(\nabla u^{(\varepsilon)}\right)\right|_{L_{p /(p-2)}(D)} \\
& \lesssim\left|a_{\varepsilon}^{\prime}\left(\nabla u_{n}^{(\varepsilon)}\right)-a_{\varepsilon}^{\prime}\left(\nabla u^{(\varepsilon)}\right)\right|_{L_{p /(p-2)}(D)} \stackrel{n \rightarrow \infty}{\longrightarrow} 0
\end{aligned}
$$

In fact, since $a_{\varepsilon}^{\prime}$ is Lipschitz, it follows that $\left|a_{\varepsilon}^{\prime}(x)\right| \lesssim|x|^{p-2}+1$, and $\nabla u_{n}^{(\varepsilon)} \rightarrow \nabla u^{(\varepsilon)}$ in $L_{p}$ implies convergence a.e. on a subsequence, from which we can conclude by 
the dominated convergence theorem (see e.g. [2, Thm. 1.2.6] for complete details in a similar situation). We have thus proved that $A^{\varepsilon} \in C^{1}\left(V \rightarrow V^{\prime}\right)$.

We conclude proving that

$$
\lim _{\varepsilon \rightarrow 0}\left|A^{\varepsilon} u-A u\right|_{V^{\prime}}=0 \quad \forall u \in V .
$$

By (26) we have

$$
\begin{aligned}
\int_{D}\left|\left\langle a_{\varepsilon}\left(\nabla u^{(\varepsilon)}\right), \nabla w^{(\varepsilon)}\right\rangle-\langle a(\nabla u), \nabla w\rangle\right| d \xi \\
\quad \leq \int_{D}\left|\left\langle a_{\varepsilon}\left(\nabla u^{(\varepsilon)}\right)-a(\nabla u), \nabla w\right\rangle\right| d \xi+\int_{D}\left|\left\langle a_{\varepsilon}\left(\nabla u^{(\varepsilon)}\right), \nabla w^{(\varepsilon)}-\nabla w\right\rangle\right| d \xi .
\end{aligned}
$$

Since $\left|a_{\varepsilon}(x)\right| \leq N\left(|x|^{p-1}+1\right)$ with $N$ independent of $\varepsilon$, the second term on the right-hand side can be majorized by

$$
\left|a_{\varepsilon}\left(\nabla u^{(\varepsilon)}\right)\right|_{L_{q}}\left|\nabla w^{(\varepsilon)}-\nabla w\right|_{L_{p}} \lesssim\left(|\nabla u|_{L_{p}}^{p-1}+1\right)\left|\nabla w^{(\varepsilon)}-\nabla w\right|_{L_{p}} \stackrel{\varepsilon \rightarrow 0}{\longrightarrow} 0
$$

where we have used once again Lemma 4.1. Since $\nabla u^{(\varepsilon)} \rightarrow \nabla u$ in $L_{p}$ as $\varepsilon \rightarrow 0$, we can upgrade the convergence to a.e. convergence, passing to a subsequence, still denoted by $\varepsilon$. By Egorov's theorem, there exists $D_{\delta} \subset D,\left|D \backslash D_{\delta}\right| \leq \delta$, such that $\nabla u^{(\varepsilon)} \rightarrow \nabla u$ uniformly on $D_{\delta}$ as $\varepsilon \rightarrow 0$. Since $a_{\varepsilon}$ and its limit function $a$ are continuous on $\mathbb{R}^{d}$, we have

$$
\lim _{\varepsilon \rightarrow 0} \lim _{\eta \rightarrow 0} a_{\varepsilon}\left(\nabla u^{(\eta)}\right)=\lim _{\eta \rightarrow 0} \lim _{\varepsilon \rightarrow 0} a_{\varepsilon}\left(\nabla u^{(\eta)}\right)=a(\nabla u)
$$

pointwise on $D_{\delta}$, hence by a diagonal extraction argument, there exists a further subsequence of $\varepsilon$, still denoted by $\varepsilon$, such that, by the dominated convergence theorem,

$$
\left|a_{\varepsilon}\left(\nabla u^{(\varepsilon)}\right)-a(\nabla u)\right|_{L_{q}\left(D_{\delta}\right)} \stackrel{\varepsilon \rightarrow 0}{\longrightarrow} 0 .
$$

On the other hand, we have

$$
\begin{aligned}
\left|a_{\varepsilon}\left(\nabla u^{(\varepsilon)}\right)-a(\nabla u)\right|_{L_{q}\left(D \backslash D_{\delta}\right)} & \lesssim \int_{D \backslash D_{\delta}}\left(|\nabla u|^{p}+1\right) d \xi \\
& \lesssim\left|D \backslash D_{\delta}\right|\left(|\nabla u|_{L_{p}}+1\right) \leq \delta\left(|\nabla u|_{L_{p}}+1\right) .
\end{aligned}
$$

Since $\delta$ is arbitrary, we conclude that the integral above converges to zero as $\varepsilon \rightarrow 0$, thus finishing the proof.

4.3. Stochastic porous media equations. Existence and uniqueness of invariant measures, their ergodicity, and Kolmogorov equations for stochastic porous media equations perturbed by a Wiener noise have been considered in several recent papers - see e.g. $[\mathbf{7}, \mathbf{1 0}]$ and references therein. Here we show how our abstract results can be applied to a class of porous media equations perturbed by general additive noise obtained as integral with respect to a compensated Poisson measure.

Let $D, \Delta, p, q$, and $\left\{\zeta_{\varepsilon}\right\}$ be defined as in the previous subsection. Set $V=$ $L_{p}(D), H=W_{2}^{-1}(D), V^{\prime}=\Delta\left(L_{q}(D)\right)$, so that $V \hookrightarrow H$ compactly by a Sobolev embedding theorem (see e.g. [27, Prop. 4.6]). The norm in $W_{2}^{-1}(D)$ will be denoted by $|\cdot|_{-1}$. Consider the operator

$$
\begin{aligned}
A: V & \rightarrow V^{\prime} \\
u & \mapsto-\Delta \beta(u),
\end{aligned}
$$


where $\beta \in C^{0}(\mathbb{R})$ is increasing and satisfies

$$
x \beta(x) \gtrsim|x|^{p}-1, \quad|\beta(x)| \lesssim|x|^{p-1}+1
$$

for all $x \in \mathbb{R}$. Note that these conditions on $\beta$ imply that $A$ is well-defined (see e.g. $[\mathbf{2 4}, \S 4.1]$ for details). Set

$$
\beta_{\varepsilon}(x)=\tilde{\beta}_{\varepsilon} * \zeta_{\varepsilon}, \quad \tilde{\beta}_{\varepsilon}=-\varepsilon^{-1} \vee \beta(x) \wedge \varepsilon^{-1},
$$

so that $\beta_{\varepsilon} \in C_{b}^{\infty}$, and define the operator

$$
A^{\varepsilon} u:=-\Delta(I-\varepsilon \Delta)^{-1} \beta_{\varepsilon}\left((I-\varepsilon \Delta)^{-1} u\right)
$$

on smooth functions. Then $A^{\varepsilon}$ is well-defined as an operator from $H$ to itself, since

$$
\left\langle A^{\varepsilon} u, w\right\rangle_{-1}=\int_{D} \beta_{\varepsilon}\left(u^{(\varepsilon)}\right) w^{(\varepsilon)} d \xi \leq\left|w^{(\varepsilon)}\right|_{L_{2}(D)}\left|\beta_{\varepsilon}\left(u^{(\varepsilon)}\right)\right|_{L_{2}(D)} \lesssim \varepsilon|w|_{-1}\left(|u|_{-1}+1\right)
$$

for all $u, w \in W_{2}^{-1}(D)$, because $\beta_{\varepsilon}$ is Lipschitz and $\left|u^{(\varepsilon)}\right|_{L_{2}(D)} \lesssim|u|_{-1}$ (see e.g. [5, Thm. 3.3.1]). A completely analogous computation also shows that $A^{\varepsilon} \in \dot{C}^{0,1}(H \rightarrow$ $H)$. Let us also show that $A^{\varepsilon}$ is well-defined as an operator from $V$ to $V^{\prime}$ : for $u$, $w \in L_{p}(D)$, Hölder's inequality yields

$$
\left\langle A^{\varepsilon} u, w\right\rangle=\int_{D} \beta_{\varepsilon}\left(u^{(\varepsilon)}\right) w^{(\varepsilon)} d \xi \leq\left|w^{(\varepsilon)}\right|_{L_{p}(D)}\left|\beta_{\varepsilon}\left(u^{(\varepsilon)}\right)\right|_{L_{q}(D)} \lesssim|w|_{L_{p}(D)}\left(|u|_{L_{p}(D)}+1\right),
$$

where we have used Lemma 4.1 and the estimate $\left|\beta_{\varepsilon}(x)\right| \leq|\beta(x)| \lesssim|x|^{p-1}+1$. The latter also immediately implies that $\left|A^{\varepsilon} x\right|_{V^{\prime}} \leq N\left(|x|_{V}^{p-1}+1\right)$, with $N$ independent of $\varepsilon$.

As in the previous subsection, it is not difficult to see that $A^{\varepsilon}$ is Gâteaux differentiable from $V$ to $V^{\prime}$, with Gâteaux differential

$$
\left\langle\left(A^{\varepsilon}\right)^{\prime} u[v], w\right\rangle=\int_{D} \beta_{\varepsilon}^{\prime}\left(u^{(\varepsilon)}\right) v^{(\varepsilon)} w^{(\varepsilon)} d \xi, \quad u, v, w \in L_{p}(D) .
$$

The continuity of the Gâteaux differential (hence the Fréchet differentiability of $A^{\varepsilon}: V \rightarrow V^{\prime}$ ) follows by an argument similar to the one used in the previous subsection, and we shall be more concise here: for $u_{n} \rightarrow u$ in $L_{p}(D)$, we have

$$
\begin{aligned}
\left\langle\left(A^{\varepsilon}\right)^{\prime}\left(u_{n}\right)[v]-\left(A^{\varepsilon}\right)^{\prime}(u)[v], w\right\rangle & \leq\left|w^{(\varepsilon)}\right|_{L_{p}(D)}\left|\left[\beta_{\varepsilon}^{\prime}\left(u_{n}^{(\varepsilon)}\right)-\beta_{\varepsilon}^{\prime}\left(u^{(\varepsilon)}\right)\right] v^{(\varepsilon)}\right|_{L_{p /(p-1)}(D)} \\
& \lesssim|v|_{L_{p}(D)}|w|_{L_{p}(D)}\left|\beta_{\varepsilon}^{\prime}\left(u_{n}^{(\varepsilon)}\right)-\beta_{\varepsilon}^{\prime}\left(u^{(\varepsilon)}\right)\right|_{L_{p /(p-2)}(D)} .
\end{aligned}
$$

We proceed now as above: since $u_{n}^{(\varepsilon)} \rightarrow u^{(\varepsilon)}$ a.e. along a subsequence, we can appeal to the dominated convergence theorem, in view of the obvious bound $\left|\beta_{\varepsilon}^{\prime}(x)\right| \lesssim$ $|x|^{p-2}+1$.

The proof that $A^{\varepsilon} u \rightarrow A u$ in $V^{\prime}$ for all $u \in V$ as $\varepsilon \rightarrow 0$ is completely similar to the corresponding proof in the previous subsection, hence omitted.

\section{References}

1. C. D. Aliprantis and K. C. Border, Infinite dimensional analysis, third ed., Springer, Berlin, 2006. MR MR2378491

2. A. Ambrosetti and G. Prodi, A primer of nonlinear analysis, Cambridge University Press, Cambridge, 1995. MR MR1336591 (96a:58019)

3. E. Asplund, Averaged norms, Israel J. Math. 5 (1967), 227-233. MR MR0222610 (36 \#5660)

4. V. Barbu, Nonlinear semigroups and differential equations in Banach spaces, Noordhoff, Leyden, 1976. MR MR0390843 (52 \#11666) 
5. - Partial differential equations and boundary value problems, Kluwer Academic Publishers, Dordrecht, 1998. MR MR1636579 (2000e:35002)

6. V. Barbu and G. Da Prato, Ergodicity for nonlinear stochastic equations in variational formulation, Appl. Math. Optim. 53 (2006), no. 2, 121-139. MR MR2172782 (2007d:60030)

7. V. I. Bogachev, G. Da Prato, and M. Röckner, Invariant measures of generalized stochastic equations of porous media, Dokl. Akad. Nauk 396 (2004), no. 1, 7-11. MR MR2115900 (2005k:60191)

8. G. Da Prato, Kolmogorov equations for stochastic PDEs, Birkhäuser Verlag, Basel, 2004. MR MR2111320 (2005m:60002)

9. Kolmogorov equations for stochastic PDE's with multiplicative noise, Stochastic analysis and applications, Abel Symp., vol. 2, Springer, Berlin, 2007, pp. 235-263. MR MR2397790 (2010a:60216)

10. G. Da Prato, M. Röckner, B. L. Rozovskii, and Feng-Yu Wang, Strong solutions of stochastic generalized porous media equations: existence, uniqueness, and ergodicity, Comm. Partial Differential Equations 31 (2006), no. 1-3, 277-291. MR MR2209754 (2007b:60153)

11. J. Dieudonné, Foundations of modern analysis, Academic Press, New York, 1960. MR 22 \#11074

12. I. Gyöngy, On stochastic equations with respect to semimartingales. III, Stochastics 7 (1982), no. $4,231-254$.

13. I. Gyöngy and N. V. Krylov, On stochastic equations with respect to semimartingales. I, Stochastics 4 (1980/81), no. 1, 1-21. MR MR587426 (82j:60104)

14. - On stochastics equations with respect to semimartingales II. Itô formula in Banach spaces, Stochastics 6 (1981/82), no. 3-4, 153-173. MR MR665398 (84m:60070a)

15. N. Ikeda and S. Watanabe, Stochastic differential equations and diffusion processes, second ed., North-Holland Mathematical Library, vol. 24, North-Holland Publishing Co., Amsterdam, 1989. MR MR1011252 (90m:60069)

16. N. V. Krylov, Lectures on elliptic and parabolic equations in Sobolev spaces, American Mathematical Society, Providence, RI, 2008. MR MR2435520

17. N. V. Krylov and B. L. Rozovski1̆, Stochastic evolution equations, Current problems in mathematics, Vol. 14 (Russian), Akad. Nauk SSSR, Vsesoyuz. Inst. Nauchn. i Tekhn. Informatsii, Moscow, 1979, pp. 71-147, 256. MR MR570795 (81m:60116)

18. C. Marinelli and M. Röckner, Well-posedness and ergodicity for stochastic reaction-diffusion equations with multiplicative Poisson noise, 2009, arXiv:0903.3299.

19. M. Métivier, Semimartingales, Walter de Gruyter \& Co., Berlin, 1982. MR MR688144 (84i:60002)

20. V. Nersesyan, Polynomial mixing for the complex Ginzburg-Landau equation perturbed by a random force at random times, J. Evol. Equ. 8 (2008), no. 1, 1-29. MR MR2383481 (2009d:35314)

21. E. Pardoux, Equations aux derivées partielles stochastiques nonlinéaires monotones, Ph.D. thesis, Université Paris XI, 1975.

22. A. Pazy, Semigroups of linear operators and applications to partial differential equations, Springer-Verlag, New York, 1983. MR 85g:47061

23. Sz. Peszat and J. Zabczyk, Stochastic partial differential equations with Lévy noise, Cambridge University Press, Cambridge, 2007. MR MR2356959

24. C. Prévôt and M. Röckner, A concise course on stochastic partial differential equations, Lecture Notes in Mathematics, vol. 1905, Springer, Berlin, 2007. MR MR2329435

25. A. V. Skorokhod, Asymptotic methods in the theory of stochastic differential equations, "Naukova Dumka", Kiev, 1987. MR MR913305 (88m:60164)

26. W. Stannat, (Nonsymmetric) Dirichlet operators on $L^{1}$ : existence, uniqueness and associated Markov processes, Ann. Scuola Norm. Sup. Pisa Cl. Sci. (4) 28 (1999), no. 1, 99-140. MR MR1679079 (2000b:31010)

27. H. Triebel, Theory of function spaces. III, Birkhäuser Verlag, Basel, 2006. MR MR2250142 (2007k:46058) 
(C. Marinelli) Facoltà di Economia, Università di Bolzano, Piazza Università 1, I39100 Bolzano, Italy and Dipartimento di Matematica, Università di Trento, via SomMARIVE 14, I-38123 TRENTo, ItALy. HTtP://WWW.Uni-BonN.DE/ CM788

E-mail address: carlo.marinelli@unibz.it

(G. Ziglio) Dipartimento di Matematica, Università di Trento, via Sommarive 14, I-38123 Trento, ItALY

E-mail address: ziglio@science.unitn.it 